\title{
Supergravity Unification
}

\author{
Ali H. Chamseddine ${ }^{\mathrm{a}}$, R. Arnowitt ${ }^{\mathrm{b}}$ and Pran Nath ${ }^{\mathrm{c}} \mathrm{d} *$

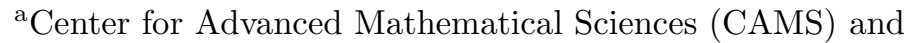 \\ Physics Department, American University of Beirut, Lebanon \\ ${ }^{\mathrm{b}}$ Center for Theoretical Physics, Department of Physics, Texas A\& M University, College Station TX \\ $77843,-4242, \mathrm{USA}$ \\ ${ }^{\mathrm{c}}$ Theoretical Physics Division, CERN CH-1211, Geneve 23, Switzerland \\ d Department of Physics, Northeastern University, Boston, MA 02115-5000, USA
}

A review is given of the historical developments of 1982 that lead to the supergravity unified model (SUGRA) with gravity mediated breaking of supersymmetry. Further developments and applications of the model in the period 1982-85 are also discussed. The supergravity unified model and its minimal version (mSUGRA) are currently among the leading candidates for physics beyond the Standard Model. A brief note on the developments from the present vantage point is included.

\section{Introduction}

The main advantage of supersymmetry [1] in the context of building models of particle interactions is that it offers a technical solution to the hierarchy problem of masses [2]. Thus, for example, the Higgs field is not protected from acquiring large masses though loop corrections and in a grand unified theory the radiative corrections would give it a GUT size mass of $\mathrm{O}\left(10^{14}\right) \mathrm{GeV}$. However, if the scalar fields appear in a supermultiplet then supersymmetry would require a cancellation of the loop corrections in the supersymmetric limit. When supersymmetry is broken the loop corrections to the Higgs mass and to other masses will then have a dependence on the SUSY breaking scale. So one finds that the breaking of supersymmetry is central to getting a meaningful result out of supersymmetric theories. Up to 1982 models in particle physics were in the framework of global supersymmetry[3]. However, this effort faced an important hurdle, i.e., it is difficult to break global supersymmetry in a phenomenologically acceptable fashion. Thus

\footnotetext{
*Permanent address of P.N
}

efforts to break global supersymmetry spontaneously lead to some unpleasant features as discussed below and consequently such efforts were abondoned. An alternative suggestion, advanced however, was to introduce in an ad hoc fashion soft breaking terms consisting of dimension 2 and dimension 3 operators in the theory which break supersymmetry but nonetheless respect the ultraviolet behavior of the theory and respect the gauge hierarchy since such terms are super renormalizable [4.5]. The list of such soft terms consists of scalar mass terms such as for squarks and sleptons and for the Higgs, bilinear scalar terms, Majorana mass terms for the gauginos, as well as cubic bosonic terms involving products of scalar fields. However, such a procedure introduces generally an enormous number of free parameters in the theory. For the so called Minimal Supersymmetric Standard Model this number is around 104. A theory such as this is not predictive.

To understand the origin of soft breaking terms and to derive them from the basic model one needs to break supersymmetry spontaneously. However, the spontaneous breaking of supersymmetry leads to a Goldstone fermion, or a Goldstino and it is important to absorb this Goldstino 
to achieve a meaningful theory which is not in contradiction with experiment. To absorb the Goldstino one needs a vector-spinor, i.e., a massless spin $3 / 2$ field or a gravitino. The necessity to get rid of the undesirable Goldstino requires that we consider an extension of supersymmetry to include gravity. In this extension the gravitino is the superparter of the graviton and thus one is led to consider supergravity [6] as the starting point for a phenomenologically viable breaking of supersymmetry. However, to build models within supergravity one needs to couple $\mathrm{N}=1$ supergravity with chiral multiplets and a vector multiplet leading to the so called applied supergravity [7, \&]. Remarkably the framework of applied supergravity allows one to break supersymmetry in a spontaneous fashion and also to build realistic models. One of the basic ingredients in the analysis is that the scalar potential of the theory is not positive definite which was one of the main hurdles in finding a global vacuum with broken supersymmetry in the framework of global supersymmetry. For the case of supergravity grand unification proposed in 1982 [9] it is possible to break supersymmetry spontaneously in a so called hidden sector and arrange the vacuum energy to vanish. The information of supersymmetry breaking from the hidden sector to the physical sector is communicated by gravity resulting in the growth of soft breaking terms in the physical sector 9,10]. For definiteness the GUT group chosen in the 1982 work was $\mathrm{SU}(5)$ although the main results of the effective theory are not tied to any specific GUT group 9, 11, 12].

Supergravity grand unification model proposed in 1982 also resolves another problem, i.e., the puzzle of the breaking of the electro-weak $S U(2)_{L} \times U(1)_{Y}$ symmetry. There is no natural mechanism for the breaking of the electro-weak symmetry in the Standard Model, and one arranges for the breaking by the introduction of a tachyonic mass term. However, the introduction of such a tachyonic mass term is rather ad hoc. Supergravity grand unification provides a natural mechanisms for the generation of the tachyonic mass term. In SUGRA unification one starts at the GUT scale with prescribed boundary conditions on the gauge coupling constants, Yukawa couplings and on the soft parameters. The evolution of these parameters from the GUT scale down to the electro-weak scale turns the determinant of the Higgs mass square matrix negative which triggers the breaking of the electro-weak symmetry [13]. In the framework of MSSM there are two Higgs doublets in the theory, one which gives mass to the up quark and the other which gives mass to the down quark. These two doublets provide a bilinear term with a mixing parameter $\mu$ in the superpotential. Although this term is supersymmetric the $\mu$ parameter is of the size of the soft breaking terms. An important aspect of the radiative breaking of the electroweak symmetry is that the spontaneous symmetry breaking conditions allow one to determine the $\mu$ parameter and this eliminates one of the arbitrary parameters in the theory.

The radiative breaking of the electro-weak symmetry which arises in SUGRA model relates the weak scale $M_{W}$ with the soft SUSY breaking scale which in turn depends on the Planck constant. The SUGRA model in this fashion connects two fundamental scales of physics, i.e., the Fermi scale and the Planck scale. Further, the fact that $M_{W}$ is determined in terms of soft parameters up to gauge and Yukawa coupling constant factors means that the soft parameters must lie in the $\mathrm{TeV}$ range, or else, one is faced with a new kind of a fine tuning problem. This result leads to the remarkable prediction in SUGRA GUTS that one must have sparticles lying in the $\mathrm{TeV}$ mass range.

\section{SUGRA Unification}

We discuss now the main features of the work of Ref.[9]. To build models containing gravity one needs to couple supergravity with an arbitrary number of chiral multiples $\left\{\chi_{i}(x), z_{i}(x)\right\}$ (where $\chi_{i}(x)$ are left $(\mathrm{L})$ Weyl spinors and $z_{i}(x)$ are scalar fields) and a vector multiplet which belongs to the adjoint representation of the gauge group. Such a coupling scheme depends on three arbitrary functions: : the superpotential $g\left(z_{i}\right)$, the gauge kinetic function $f_{\alpha \beta}\left(z_{i}\right)$ (which enters in the Lagrangian as $f_{\alpha \beta} F_{\mu \nu}^{\alpha} F^{\mu \nu \beta}$ with $\alpha, \beta=$ gauge indices) and the Kahler potential $d\left(z_{i}, z_{i}^{\dagger}\right)$. The 
bosonic part of the Lagrangian is given by

$$
\begin{array}{r}
L_{B}=-\frac{e}{2 \kappa^{2}} R+\frac{e}{\kappa^{2}} G_{, b}^{a} D_{\alpha} z_{a} D^{\alpha} z^{b} \\
-\frac{e}{4} f_{\alpha \beta} F_{\mu \nu}^{\alpha} F^{\mu \nu \beta}-e V
\end{array}
$$

Here $\kappa \equiv 1 / M_{P \ell}$ where $M_{\text {Planck }}=2.4 \times 10^{18}$ $\mathrm{GeV}, \mathrm{G}$ depends on the superpotential $\mathrm{g}(\mathrm{z})$, and on the Kahler potential $d\left(z, z^{\dagger}\right)$ so that

$G=-\frac{\kappa^{2}}{2} d\left(z, z^{\dagger}\right)-\ln \left(\frac{\kappa^{6}}{4}|g|^{2}\right)$

and $\mathrm{V}$ is the effective potential for the scalar components of the chiral multiplets in the theory and is given by

$V=-\frac{1}{\kappa^{4}} e^{-G}\left(3+G_{b}^{-1 a} G,{ }_{, a} G,{ }^{b}\right)+\frac{1}{8 \kappa^{4}}\left(G, a T^{\alpha} z^{a}\right)^{2}(3)$

As noted in the introduction the potential is no longer positive definite because of the Planck scale corrections from supergravity. Of course, in the limit $M_{\text {Planck }} \rightarrow \infty$ one recovers the result of global supersymmetry. However, the terms proportional to $1 / M_{P \text { Lanck }}$ are essential in generating an acceptable spontaneous breaking of supersymmetry. The simplest way of breaking supersymmetry spontaneously is to introduce a term which is a chiral gauge singlet and enters linearly in the superpotential, i.e.,

$g_{S B}=m^{2}(z+B)$

where the constant $\mathrm{B}$ is chosen to absorb the vanishing of the cosmological constant. This term generates a spontaneous breaking of supersymmetry and gives a gravitino mass of size $\mathrm{m}^{2} / M_{\text {Planck }}$. However, it was later realized that the precise form of the superpotential that breaks supersymmetry is not important, and essentially what one needs is any form that breaks supersymmetry and has the property that $g_{S B}=g_{2}(z)$ where 12

$g_{2}=\frac{m^{2}}{\kappa} f(\kappa z)$

so that at the minimum 12

$$
<z>\sim \frac{1}{\kappa}, \quad<\kappa z>\sim O(1), \quad<g_{2}>\simeq \frac{m^{2}}{\kappa}(6)
$$

and one has in this case that the gravitino mass is

$m_{\frac{3}{2}}=\kappa^{2}<g_{2}>=\kappa m^{2}$

In addition to the bosonic interactions one also has interaction structures in the theory which involve gauge couplings of the gauginos to the chiral multiplets and these take the form

$L_{\lambda}=-i g_{\alpha} \bar{\lambda}^{\alpha} z_{i}\left(\frac{T^{\alpha}}{2}\right)_{i j} \chi^{j}+$ h.c.

Further, the superpotential contributes to both the fermi and the bose interactions. Thus the Fermi interactions for the Weyl spinors $\chi^{i}$ are given by 14

$L_{g}=-\frac{1}{2}\left(\bar{\chi}^{i C} g_{i j} \chi^{j}+\right.$ h.c. $)$

We now elaborate on some of the further details in the construction of SUGRA GUT models. One of the immediate problems one faces in constructing such models is that of protecting the gauge hierarchy. Since $<z>\sim O\left(M_{\text {Planck }}\right)$ one finds that terms involving $z_{i} z_{j} z$ would develop masses which are size $O\left(M_{\text {Planck }}\right)$ and one has to suppress them. A very convenient way to overcome this problem is to use the separation of the hidden and the physical sectors, i.e., place the chiral multiplets of the physical sector in one part of the superpotential $\left(g_{1}\right)$ and place the chiral fields that break supersymmetry in another part of the superpotential in the hidden sector $\left(g_{2}\right)$ with no direct interaction between the two sectors, i.e., one writes 9,22

$g\left(z_{i}\right)=g_{1}\left(z_{a}\right)+g_{2}(z)$

where $\left\{z_{i}\right\}=\left\{z_{a}, z\right\}$ where $z_{a}$ are physical sector fields (squarks, sleptons, higgs) and $\mathrm{z}$ are the hidden sector fields whose VEVs $\langle z\rangle=\mathcal{O}\left(M_{P \ell}\right)$ break supersymmetry. In this arrangement of separating the superpotential into a physical sector and a hidden sector one finds that the gauge hierarchy is maintained by the additive nature of the physical and the hidden sector terms in the superpotential. The actual proof of this remarkable theorem that the low energy theory is protected from corrections proportional to the GUT 
scale and the Planck scale is rather subtle and we shall discuss this shortly. For the physical sector of the theory which involves the quarks, the leptons and the higgs we take the following potential

$$
\begin{array}{r}
g_{1}=\lambda_{1}\left(\frac{1}{3} \operatorname{tr} \Sigma^{3}+\frac{1}{2} M \operatorname{tr} \Sigma^{2}\right)+\lambda_{2} H^{\prime}\left(\Sigma+3 M^{\prime}\right) H \\
+\lambda_{3} U H^{\prime} H+\epsilon_{x y z v w} H^{x} M^{y z} f_{1} M^{v w} \\
+H_{x}^{\prime} M^{x y} f_{2} M_{y}^{\prime}
\end{array}
$$

Here $M^{x y}$ and $M_{x}^{\prime}$ are the 10 and $\overline{5}$ of quarks and leptons, $H^{\prime}$ and $H$ are the $\overline{5}$ and 5 of Higgs, $\mathrm{U}$ is a singlet and $\Sigma$ is a 24 plet of $\mathrm{SU}(5)$. Minimization of the scalar potential in this case gives the result

$$
<\Sigma_{y}^{x}>=\operatorname{MDiag}\left(2,2,2,-3+\epsilon_{2},-3-\epsilon_{2}\right)
$$

and $<H^{x}>=<H_{x}^{\prime}>\sim O\left(\kappa m^{2}\right) \delta_{5}^{x}$, where $\epsilon_{2}$ depends on the soft SUSY parameters. Here one finds quite remarkably that supersymmetry breaking in the hidden sector induces the breaking of $S U(2)_{L} \times U(1)_{Y}$ so that $S U(2)_{L} \times U(1)_{Y} \rightarrow$ $U(1)_{e m}$. One notes that the breaking of $S U(2)_{L} \times$ $U(1)_{Y}$ is semi-gravitational with $\kappa m^{2} \sim 300 \mathrm{GeV}$ and can account for the $\mathrm{W}$ and $\mathrm{Z}$ mass.

As noted earlier the miraculous thing in this model is that the integration of the heavy fields do not mix with the low energy sector of the theory. In general the presence of the large scale $M \sim M_{G}$ and $M_{\text {Planck }}$ introduces a new hierarchy problem as one can expect gravitaional corrections of sizes 9, 92

$(\kappa M) M, \quad(\kappa M)^{2} M, . .,(\kappa M)^{6} M, .$.

However, the detailed analyses show that all such corrections vanish, and the low energy theory contains only terms of $O\left(\kappa m^{2}\right)$. An analysis under the assumption of a flat Kahler potential and on integration over the heavy fields shows that the low energy theory can be parametrized by $9,11,12$ ]

$V_{\text {soft }}=m_{0}^{2} z_{a} z_{a}^{\dagger}+\left[\frac{1}{3} A^{0} W^{(3)}+\frac{1}{2} B^{0} W^{(2)}+\right.$ h.c. $]$

where $W^{(3)}$ is the cubic part and $W^{(2)}$ is the quadratic part of the superpotential. Thus one finds that the soft SUSY breaking with the assumption of the flat Kahler potential generates a universal mass for the scalar fields, and also universal bilinear and trilinear couplings arise in the theory. While in the simple model with a flat gauge kinetic energy function the gauginos are massless at the tree level they do develop masses through loop corrections where the GUT fields circulate in the loops with masses 15]

$\tilde{m}_{i}=\frac{\alpha_{i}}{4 \pi} m_{\frac{3}{2}} C \frac{D(R)}{D(A)}$

where $\alpha_{i}$ is the gauge coupling constant associated with the sub groups $S U(3), S U(2)$ and $U(1)$, and $C$ is the Casimir, $\mathrm{D}(\mathrm{R})$ is the dimensionality of the representation exchanged, and $\mathrm{D}(\mathrm{A})$ is the dimensionality of the adjoint representation. One can also generate tree level masses by assuming a field dependence in the gauge kinetic energy function $f_{\alpha \beta}$. In this case one finds that the gauginos gain a mass at $M_{G}$ of $\left(m_{1 / 2}\right)_{\alpha \beta} \lambda^{\alpha} \gamma^{0} \lambda^{\beta}\left(\lambda^{\alpha}=\right.$ gaugino Majorana field) where

$\left(m_{1 / 2}\right)_{\alpha \beta}=\frac{1}{4} \kappa^{-1}\left\langle G^{i}\left(d^{-1}\right)_{j}^{i} f_{\alpha \beta j}^{\dagger}\right\rangle m_{3 / 2}$

Here $G^{i} \equiv \partial G / \partial z_{i}^{\dagger},\left(d^{-1}\right)_{j}^{i}$ is the matrix universe of $d_{j}^{i}$ and $f_{\alpha \beta j}=\partial f_{\alpha \beta} / \partial z_{j}$. For the case of the flat Kahler potential where $d_{j}^{i}=\delta_{j}^{i}$ one finds that the gaugino masses are universal at the GUT scale. Further, more generally one can write the gravitino mass so that

$m_{\frac{3}{2}}=\kappa^{-1}\langle\exp [G / 2]\rangle$

In the above we discussed how spontaneous supersymmetry breaking in the hidden sector induces soft breaking terms in the physical sector of the theory which in turn induce breaking of the electro-weak symmetry. The fact that the spontaneous breaking of the supersymmetry triggers the breaking of the electro-weak symmetry establishes for the first time a direct connection between physics at the Planck scale and the physics at the electro-weak scale. The electroweak symmetry breaking in the model of Eq.(1) was induced at the tree level through an effective cubic interaction. Now it turns out that one can in fact do away with the cubic term 
and induce spontaneous breaking of the electroweak symmetry via renormalization group effects. The basic idea here is that the one uses the GUT boundary conditions on the gauge coupling constants and the Yukawa couplings, and the boundary conditions on the soft parameters at the GUT scale and uses the renormalization group equations to evolve them down from the GUT scale to the electro-weak scale. The running of the renormalization group equations with at least one soft SUSY breaking parameter non-zero and the top quark Yukawa coupling turn the determinant of the Higgs mass matrix (and specifically the $\mathrm{H}_{2}$ running $\left(\right.$ mass) ${ }^{2}$ ) negative which triggers the breaking of the electroweak symmetry. The minimization conditions for the scalar potential with respect to the Higgs VEVs $v_{1,2}=\left\langle H_{1,2}\right\rangle$ at the electro-weak scale provides the following two relations 13 $\mu^{2}=$ $\left(\mu_{1}^{2}-\mu_{2}^{2} \tan ^{2} \beta\right) /\left(\tan ^{2} \beta-1\right)-M_{Z}^{2} / 2 ;$ and $\sin ^{2} \beta$ $=(-2 B \mu)\left(2 \mu^{2}+\mu_{1}^{2}+\mu_{2}^{2}\right)$. Here $\tan \beta=v_{2} / v_{1}, \mathrm{~B}$ is the quadratic soft breaking parameter $\left(V_{\text {soft }}^{B}=\right.$ $\left.B \mu H_{1} H_{2}\right), \mu_{i}=m_{H_{i}}^{2}+\Sigma_{i}$, and $\Sigma_{i}$ are loop corrections. In the above all parameters are running parameters at the electroweak scale $Q$ which can be taken to be $Q=M_{Z}$. The first relation above determines $\mu$ while the second allows one to eliminate $\mathrm{B}$ in favor of $\tan \beta$. This determination increases the predictivity of the model. With inclusion of the constraints of the electro-weak symmetry breaking the minimal SUGRA model (mSUGRA) depends on four soft breaking parameters and one sign

$m_{0}, m_{1 / 2}, A_{o}, B_{0}, \operatorname{sign}(\mu)$

Alternately one may choose $m_{0}, m_{\tilde{g}}, A_{t}, \tan \beta$, and $\operatorname{sign}(\mu)$ as the independent parameters. If one allows for complex soft parameters then after field redefinitions there are two additional parameters which can be chosen to be the phase of $A_{0}$ and the phase of $\mu$. The appearance of the phases brings into the theory in a natural way new sources of $\mathrm{CP}$ violation over and above the $\mathrm{CP}$ violation in the CKM mass matrix. Further, the nature of physics at the Planck scale is not fully understood and it is likely that the assumption of a flat Kahler potential should be relaxed by the introduction of curvature terms. Such cur- vature terms sometimes appear in string models. The appearance of non-flatness in the Kahler potential generates non-universalities in the soft parameters enlarging the number of parameters in the theory 16. Further, the picture of supersymmetry breaking via chiral scalar terms in the hidden sector of the theory is an effective treatment of some more basic phenomenon in the underlying fundamental theory which is still unknown. Several possibilities for the nature of such a phenomena including the possibility of condensates breaking supersymmetry have been discussed 17 in the literature. In addition, most string models that accomodate grand unification at the GUT scale reduce to a SUGRA model below $M_{G U T}$, and thus mSUGRA remains a bench mark for testing experimental data with theory. Next we discuss the sparticle spectrum that emerges in such a theory at low energy.

The minimal SUGRA model with the soft SUSY parameters defined by Eq.(18) generates a low energy mass spectrum for the Higgs and for the sparticles which possesses identifiable properties. The Higgs sector of the theory gives three neutral fields, one of which is a pseudo-scalar (A) and the other two are scalars $(\mathrm{h}, \mathrm{H})$. The masses of the scalars are related to the masses of the pseudo-scalars by the relation [18]

$$
\begin{array}{r}
m_{h, H}^{2}=\frac{1}{2}\left[M_{Z}^{2}+M_{A}^{2} \pm\left(\left(M_{Z}^{2}+M_{A}^{2}\right)^{2}\right.\right. \\
\left.\left.-4(\cos 2 \beta)^{2} m_{Z}^{2} M_{A}^{2}\right)^{\frac{1}{2}}\right]
\end{array}
$$

In addition there is a charged Higgs boson whose mass is given by

$M_{ \pm}^{2}=M_{W}^{2}+M_{A}^{2}$

The mass relations for the scalar Higgs lead to the result 18

$m_{h} \leq M_{Z}$

Of course, this is a relation at the tree level and there are important loop corrections to this formula.

One can also discuss the masses and the couplings for the sparticles that appear in the theory. The superpartners in the theory consist of 
gauginos, Higgsinos and sfermions. The gauginoHiggsino sector involves the trilinear HiggsHiggsino-gaugino coupling which after spontaneous breaking of the electro-weak symmetry generates a gaugino -Higgsino mixing term. In the charged gaugino-Higgsino sector one finds a mass matrix (in the $\tilde{W}^{ \pm}-\tilde{H}^{ \pm}$basis) of the form 19

$M_{\chi}^{ \pm}=\left(\begin{array}{cc}\tilde{m}_{2} & \sqrt{2} M_{W} \sin \beta \\ \sqrt{2} M_{W} \cos \beta & \mu\end{array}\right)$

The eigen-vectors of this matrix (Winos) have the mass eigen-values

$\tilde{m}_{ \pm}=\frac{1}{2}\left|\left[4 \nu_{+}^{2}+\left(\mu-\tilde{m}_{2}\right)^{2}\right]^{\frac{1}{2}} \pm\left[4 \nu_{-}^{2}+\left(\mu+\tilde{m}_{2}\right)^{2}\right]^{\frac{1}{2}}\right|$

where $\tilde{m}_{ \pm}=\left|\lambda_{ \pm}\right|$and $\lambda_{ \pm}$are the eigen-values of the $\mathrm{M}$, and $\nu_{ \pm}$are defined by

$\sqrt{2} \nu_{ \pm}=M_{W}(\sin \beta \pm \cos \beta)$

In the neutral gaugino - Higgsino sector one finds (in the $\left(\tilde{W}_{3}, \tilde{B}, \tilde{H}_{1}, \tilde{H}_{2}\right)$ basis) the mass matrix

$M_{\chi}^{0}=\left(\begin{array}{cccc}\tilde{m}_{2} & o & a & b \\ o & \tilde{m}_{1} & c & d \\ a & c & o & -\mu \\ b & d & -\mu & o\end{array}\right)$

where $a=M_{Z} \cos \theta_{W} \cos \beta, c=-\tan \theta_{W} a, b=$ $-M_{Z} \cos \theta_{W} \sin \beta, \mathrm{d}=-\tan \theta_{W} b$ and $\theta_{W}$ is the weak mixing angle. The eigen-vectors of this mass matrix (Zinos) have a somewhat complicated analytical form since the eigen value equation here is a quartic one. However, the structure of the matrix reveals that there are regions where some Zinos may be mostly either gauginos or Higgsinos.

For the sfermions it is found that the masses receive contributions from the $\mathrm{D}$ terms and from the gaugino loop corrections so that at low energy one has, example for $\tilde{u}_{L}$ 19, 13,

$$
\begin{aligned}
m_{\tilde{u}_{L}}^{2}=m_{0}^{2}+m_{u}^{2} & +\tilde{\alpha}_{G}\left[\frac{8}{3} f_{3}+\frac{3}{2} f_{2}+\frac{1}{30} f_{1}\right] m_{\frac{1}{2}}^{2} \\
& +\left(\frac{1}{2}-\frac{2}{3} \sin ^{2} \theta_{W}\right) M_{Z}^{2} \cos 2 \beta
\end{aligned}
$$

and for $\tilde{u}_{R}$ one has

$$
\begin{aligned}
m_{\tilde{u}_{R}}^{2}=m_{0}+m_{u}^{2}+ & \tilde{\alpha}_{G}\left[\frac{8}{3} f_{3}+\frac{8}{15} f_{1}\right] m_{\frac{1}{2}}^{2} \\
& +\frac{2}{3} \sin ^{2} \theta_{W} M_{Z}^{2} \cos 2 \beta
\end{aligned}
$$

Here $f_{i}(t)=\left(1-1 /\left(1+\beta_{i} t\right)^{2}\right) / \beta_{i} \quad(\mathrm{i}=1,2,3)$ where $t=\ln \left(M_{G}^{2} / Q^{2}\right), \beta_{i}=\left(b_{i} / 4 \pi\right) \tilde{\alpha}_{i}$, where $b_{i}=(33 / 5,1,-3)$ for $\mathrm{U}(1), \mathrm{SU}(2)$ and $\mathrm{SU}(3)$, and $\tilde{\alpha}_{G}=\alpha_{G} /(4 \pi)$, where $f_{i}$ are the form factors. Similar relations hold for the sleptons. Using the above analysis one can write the low energy effective Lagangian in terms of the diagonalized fields. This effective Lagrangian was given in Ref. 19,8, 18].

Soon after the invention of SUGRA model there was an intense activity to elucidate its experimental implications. Since the values of $m_{0}$ and $m_{\frac{1}{2}}$ were not fixed by the theory (and remain still to be fixed by experiment) it was natural then to assume the lowest values compatible with experiment at that time. One of the important signals for SUGRA models with R parity invariance that emerged was the missing energy signal. Thus supersymmetric decays of the $\mathrm{W}$ and $\mathrm{Z}$ were widely discussed such as $W \rightarrow \tilde{W}+\tilde{Z}$ and $Z \rightarrow \tilde{W}+\tilde{Z}$ as well as decays of the Wino, the neutralino and the sfermions, etc were widely studied 20,21]

$$
\begin{array}{r}
\tilde{W} \rightarrow l+\bar{\nu}_{l}+\tilde{Z}_{i}, q_{1}+\bar{q}_{2}+\tilde{Z}_{i}, \quad h+\tilde{Z}_{i}, . . \\
\tilde{Z}_{k} \rightarrow^{ \pm}+\tilde{W}^{\mp}, \quad l+\bar{l}+\tilde{Z}_{j}, . . \\
\tilde{f} \rightarrow f+\tilde{Z}, \quad f_{1}+\tilde{W}, . .
\end{array}
$$

In all these decays the identifying signal is large missing energy associated with the disappearance of the lightest neutral particle which is absolutely stable under the assumption of $\mathrm{R}$ parity invariance. Missing energy signals at colliders also arise, such as in sparticle $(\tilde{s})$ pair production $p \bar{p} \rightarrow \tilde{s}+\tilde{s}+X$. Each sparticle is expected 
to decay producing missing energy. An important implication of the fact that the lightest supersymmetric particle (lsp) would be absolutely stable under $\mathrm{R}$ parity conservation is that such a particle is a candidate for non-baryonic cold dark matter. Specifically, the lightest neutralino appears a good candidate for the LSP 22].

Another important implication of SUGRA model that was analysed was the supersymmetric correction to the muon anomalous moment $g_{\mu}-2$. Thus soon after the formulation of the SUGRA models analyses of $g_{\mu}-2$ were carried out using the SUGRA unification framework 23]. These represented the first realistic and reliable analyses of the effects of supersymmetry on the muon anomaly. Since SUGRA provided the first realistic framework it was now possible to make quantitative predictions. Specifically it was pointed out that the effect of the electro-weak corrections could be as large or larger than the Standard Model electro-weak correction and any experiment which tests the SM electro-weak contribution to $g_{\mu}-2$ would also test the supersymmetric correction. This result played a role in the pursuit to improve the experimental limits on $g_{\mu}-2$ and provided another reason aside from checking the Standard Model result to get a better measurement when the Brookhaven experiment E821 was being proposed around 1984 .

We turn now to another aspect of SUGRA models which pertains to baryon and lepton number violation in the model. Here on must distinguish between two aspects to SUGRA unification, the first being a specific mechanism for the spontaneous breaking of supersymmetry in the hidden sector and its transmittal to the visible sector and the second being the GUT structure of the theory. The first aspect of the theory, i.e., growth of soft breaking terms protects baryon and lepton number conservation under the constraint of $\mathrm{R}$ parity invariance. Further, under the constraint of R parity invariance baryon and lepton number violating dimension four operators are also absent which eliminates rapid proton decay. However, even under the constraint of R parity one can generate dimension five operators which violate baryon and lepton number and lead to nucleon instability 24]. However, the nature of the dimension five operator depends on the nature of the GUT group and there are several possibilities here such as $\mathrm{SU}(5), \mathrm{SO}(10), \mathrm{E}(6)$ etc. Much of the early work in proton stability was in the framework of the minimal $\mathrm{SU}(5)$ model since such a group also appears in the breaking of larger GUT groups. However, what is unique about proton decay via dimension five operators is that it depends on both the GUT structure which generates the baryon and lepton number violation in the theory and on the soft breaking sector of the theory which enters in the dressing of the dimension five operators and leads to dimension six operators after dressing with the chiral structure LLLL, LLRR, RRLL and RRRR for the four fermi interaction that governs proton decay. Thus after the formulation of the SUGRA models it was possible to carry out detailed analyses of proton decay amplitudes using the framework of SUGRA unification in the dressing loop diagrams. This analysis was carried out in Refs. 25,26. As is evident predictions of the proton lifetime depend on the low energy parameters and on the GUT parameters. If one has sufficient information on the low energy parameters then that would lead to a constraint on the nature of GUT physics.

\section{A View from the Present}

In the intervening period since 1982-85 -present there has been considerable further activity in the applications of SUGRA models. Further, there is now beginning to accumulate some indirect evidence that generally favors SUGRA unification. Recall that in Sec.2 one discussed a crucial aspect of SUGRA unification which is the connection between the soft breaking scale and the electro-weak scale. Because of this connection one finds that the sparticle masses should be in the $\mathrm{TeV}$ range. This result finds an indirect support in the high precision LEP data. Thus the LEP data measures the gauge coupling constants $\alpha_{1}, \alpha_{2}$ and $\alpha_{3}$ to a great precision. Extrapolation of these coupling constants to high scales shows that they do not meet at a point using the Standard Model spectrum but do meet in MSSM with the supersymmetric particle spectrum with sparticle masses typically in the $\mathrm{TeV}$ range. The crucial ingredient in the unification of the gauge couplings is the 
fact that the SUSY particles with masses in the $\mathrm{TeV}$ range give just the right amount of corrections to the beta functions for all the three couplings to meet at a point. Thus one of the predictions of SUGRA GUTs that sparticle masses must lie in the $\mathrm{TeV}$ range finds support in the high precision LEP data. The theoretical analyses using SUGRA unification produce a unification of the gauge couplings to within 2 sigma. However, there could be additional contributions due to Planck scale corrections, which induce terms via corrections to the gauge kinetic energy term.

Since the early work of 1982-1985 the experimental lower limits on the sparticles have moved up eliminating many of the possible decay channels such as the supersymmetric decays of the $\mathrm{W}$ and Z. However, the off shell decay of particles such as the decay $W^{*} \rightarrow \tilde{W}+\tilde{Z}_{2} \rightarrow l_{1}+l_{1}+l_{2}+E_{T}$ produces a trileptonic signal which is one of the prime signals for the discovery of Winos in the Tevatron RUNII and at the LHC. Further, there is now a considerable body of work which analyses the signals in the SUGRA models in an array of channels depending on the nature of the sparticles produced (see, eg., SUGRA Working Group Collaboration, hep-ph/0003154). A very encouraging sign for weak scale supersymmetry is the recent precise determination of the muon g-2 at Brookhaven (H.N. Brown et.al., Muon (g2) Collaboration, hep-ex/0102017). The experiment finds a difference at the 2.6 sigma level between the experimental value of $a_{\mu}$ (where $a_{\mu}=$ $\left.\left(g_{\mu}-2\right) / 2\right)$ and its prediction in the Standard Model so that $a_{\mu}^{e x p}-a_{\mu}^{S M}=43(16) \times 10^{-10}$. This result indicates a very significant correction to the Standard Model from new physics. Now the prime candidate for this new physics is supersymmetry and specifically we note at this point that in the well motivated SUGRA model the supersymmetric electro-weak correction was predicted to be of the size of the Standard Model electroweak correction or larger 23. Further, analysis of this correction within mSUGRA shows that the Brookhaven result implies the existence of some low lying particles which can be produced at the LHC and possibly in RUNII of the Tevatron. Test of this prediction await experiments at RUNII and at the LHC.

\section{Conclusion}

Supergravity unification proposed 19 years ago remains one of the leading candidates for physics beyond the Standard Model. The model is consistent with all known data and predicts new physics within reach of accelerators, specifically the LHC and also possibly within reach of RUNII of the Tevatron. Deviations from the Standard Model are determined by the soft breaking sector of the theory which is governed by Planck scale physics. Thus observation of deviations from the Standard model will provide information about the nature of physics at the Planck scale and possibly about the nature of the underlying string model.

Acknowledgemnets: This work was supported in part by NSF grant numbers PHY-0070964 and PHY-9901057.

\section{References}

[1] Yu A. Golfand and E.P. Likhtman, JETP Lett. 13, 452 (1971); D. Volkov and V.P. Akulov, JETP Lett. 16, 438 (1972).

[2] S. Weinberg, in Proc. of Gauge Theories and Modern Field Theory, edit by R. Arnowitt and P. Nath, MIT Press (1975); E. Gildener, Phys. Rev. D14, 1667 (1976).

[3] J. Wess and B. Zumino, Nucl. Phys. B78, 1 (1974); S. Ferrara and B. Zumino, Nucl. Phys. B79, 413(1974); A. Salam and J. Strathdee, Fort. der Phys. 26, 57(1978).

[4] S. Dimopoulos and H. Georgi, Nucl. Phys. B193, 150 (1981); Sakai, Z. Phys. C11, 153 (1981).

[5] L. Giradello and M.T. Grisaru, Nucl. Phys. B194, 65 (1982).

[6] D.Z. Freedman, P. van Nieuwenhuizen and S. Ferrara, Phys. Rev. D14, 912 (1976); S. Deser and B. Zumino, Phys. Lett. B62, 335 (1976).

[7] E. Cremmer, S. Ferrara, L.Girardello and A. van Proeyen, Phys. Lett. 116B, 231(1982); A.H. Chamseddine, R. Arnowitt and P. Nath, Phys. Rev. Lett. 29, 970 (1982); J. Bagger and E. Witten, Phys. Lett. B118, 103(1982). 
[8] P. Nath, R. Arnowitt and A.H. Chamseddine, "Applied N =1 Supergravity" (World Scientific, Singapore, 1984).

[9] A.H. Chamseddine, R. Arnowitt and P. Nath, Phys. Rev. Lett. 29, 970 (1982).

[10] R. Barbieri, S. Ferrara and C.A. Savoy, Phys. Lett. B119, 343 (1982).

[11] L. Hall, J. Lykken and S. Weinberg, Phys. Rev. D27, 2359 (1983).

[12] P. Nath, R. Arnowitt and A.H. Chamseddine, Nucl. Phys. B227, 121 (1983).

[13] K. Inoue et al., Prog. Theor. Phys. 68, 927 (12982); L. Ibañez and G.G. Ross, Phys. Lett. B110, 227 (1982); L. Ibanez, Nucl Phys. B218, 514(1983); J. Ellis, D.V. Nanopoulos, and K. Tamvakis, Phys. Lett. B121, 123(1983); L. Alvarez-Gaumé, J. Polchinski and M.B. Wise, Nucl. Phys. B221, 495 (1983); J. Ellis, J. Hagelin, D.V. Nanopoulos and K. Tamvakis, Phys. Lett. B125, 2275 (1983); L. E. Ibañez and C. Lopez, Phys. Lett. B128, 54 (1983); Nucl. Phys. B233, 545 (1984); L.E. Ibañez, C. Lopez and C. Muñoz, Nucl. Phys. B256, 218(1985).

[14] A. Salam and J. Strathdee, Fort. der Phys. 26, 57(1978).

[15] R. Arnowitt, A.H. Chamseddine and P. Nath, in Proc. of Workshop on Problems in Unification and Supergravity, La Jolla Institute, Jan. 1983; See also Alvarez-Gaume et.al. in Ref.13.

[16] S. Soni and A. Weldon, Phys. Lett. B126, 215 (1983).

[17] H.P. Nilles, Phys. Lett. B217, 366(1983); H.P. Nilles, M. Srednicki and D. Wyler, Phys. Lett. B120, 346(1983).

[18] P. Nath, R. Arnowitt and A.H. Chamseddine, in Proceedings of the Winter School on Supersymmetry, Supergravity/Nonperturbative $Q C D$, edited by P. Roy and V. Singh (Springer, Berlin 1984),pp. 113-185.
[19] P. Nath, R. Arnowitt and A.H. Chamseddine, HUTP-83/A077, NUB No. 2588 (1983).

[20] S. Weinberg, Phys. Rev. Lett. 50, 387(1983).

[21] R. Arnowitt, A.H. Chamseddine and P. Nath, Phys. Rev. Lett. 50, 232(198); Phys. Lett. 129B, 445(1983); D.A. Dicus, S. Nandi, W.W. Repko and X.Tata, Phys. Lett. 129B, 451(1983); J. Ellis, J.S. Hagelin, D.V. Nanopoulos, and M. Srednicki, Phys. Rev. Lett. 127B, 233(1983); B. Grinstein, J. Polchinski and M. Wise, Phys. Lett. B130, 285(1983); J.M. Frere and G.L. Kane, Nucl. Phys. B223, 33(1983); M. Gluck and E. Reya, Phys. Rev. Lett. 51, 867(1983); E. Reya and D.P. Roy, Phys. Lett.B141, 442(1984).

[22] H. Goldberg, Phys. Rev. Lett. 50, 1419(1983); J. Ellis, J.S. Hagelin, D.V. Nanopoulos, K. Olive, and M. Srednicki, Nucl. Phys. B23, 453(1984).

[23] T. C. Yuan, R. Arnowitt, A.H. Chamseddine and P. Nath, Z. Phys. C26, 407(1984); D. A. Kosower, L. M. Krauss, N. Sakai, Phys. Lett. 133B, 305(1983).

[24] S. Weinberg, Phys.Rev.D26,287(1982); N. Sakai and T. Yanagida, Nucl. Phys. B197, 533(1982); S. Dimopoulos, S. Raby and F. Wilczek, Phys.Lett. 112B, 133(1982); J. Ellis, D.V.

Nanopoulos and S. Rudaz, Nucl.Phys. B202,43(1982); B.A. Campbell, J. Ellis and D.V. Nanopoulos, Phys.Lett.141B,299(1984); S. Chadha, G.D. Coughlan, M. Daniel and G.G. Ross, Phys. Lett.149B,47(1984).

[25] R.Arnowitt, A.H.Chamseddine and P.Nath, Phys.Lett. 156B,215(1985).

[26] P.Nath, R.Arnowitt and A.H.Chamseddine, Phys.Rev.32D,2348(1985). 